Check for updates

Cite this: RSC Adv., 2017, 7, 50688

\title{
Investigation of the crystallization behaviors in a sub-micron space using carbon nanocones $\uparrow$
}

\begin{abstract}
Lele Xu, ${ }^{\text {ab }}$ Yifei Ge, ${ }^{\text {bc }}$ Jianbo Liang, ${ }^{c}$ Jianxun Xu (D)*b and Xing Lu (D) *a
Up to now, there have been many research studies that have revealed the unique phenomena of various materials encapsulated in a confined nano-sized space, such as in the interior of carbon nanotubes (CNTs). Confined in such a space several nanometers in size, these materials showed many different and interesting properties. However, the morphology and behaviors of these materials encapsulated in a comparatively bigger space, i.e., from tens of nanometers to microns, have been little investigated. In this work, carbon nanocones (CNCs), which are multilayered graphene-like nanomaterials with a hollow conical structure and an inner size varying continuously from several nanometers to micrometers, were used as the workhorse to investigate the nano-confinement effects on different scales. Gd acetate was adopted to be incorporated into the conical inner space of the CNCs. The corresponding Gd oxide, formed upon heating, showed a unique morphology and crystallization behavior in this sub-micron space. The confined Gd oxide was conical in shape, perfectly fitting the interior of the CNCs. Several crystallization orientations were often presented in the Gd oxide cone structure, and single crystallization was also observed located close to the apex of the CNCs. The crystal size of Gd oxide was over $100 \mathrm{~nm}$, implying the effective scale of the confinement phenomenon between the CNCs and Gd oxide. Moreover, we further found that the Gd oxide cone retained its structure, instead of forming a spherical particle when it moved outward from the CNC driven by a high temperature, indicating the high stability of the conical Gd oxide nanocrystals.
\end{abstract}

Received 28th September 2017 Accepted 18th October 2017

DOI: 10.1039/c7ra10718h

rsc.li/rsc-advances

\section{Introduction}

Carbon materials, such as carbon nanotubes (CNTs), ${ }^{1}$ singlewalled carbon nanohorns (SWCNHs) ${ }^{2}$ and graphene, ${ }^{3}$ are widely used in various fields due to their remarkable physical and chemical properties. CNTs and SWCNHs can be seen as rolls of single-sheet graphene that have a tubular structure. Taking advantage of the hollow inner spaces in these tubular structures, various kinds of materials have been reported to be incorporated inside CNTs and SWCNHs, such as $\mathrm{H}_{2},{ }^{4,5} \mathrm{C}_{60},{ }^{6} \mathrm{Gd}$ compounds ${ }^{7}$ and cisplatin (an anti-cancer drug). ${ }^{8}$ Therefore, CNTs and SWCNHs are potentially useful for various purposes, such as gas storage, catalyst support and drug carriers. Up to now, researchers have conducted many studies on encapsulation using CNTs and SWCNHs and reported the unique behaviors of

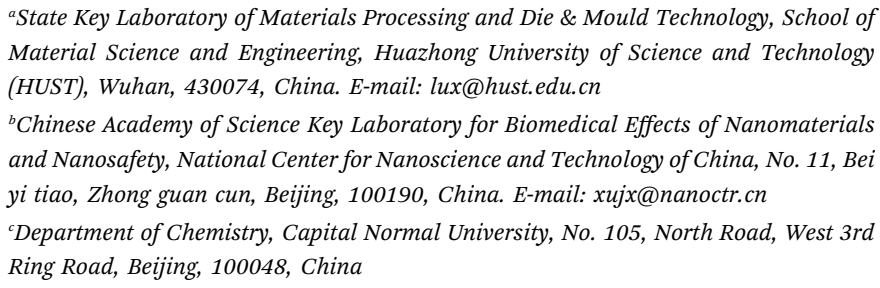

$\dagger$ Electronic supplementary information (ESI) available. See DOI: $10.1039 / \mathrm{c} 7 \mathrm{ra} 10718 \mathrm{~h}$ the conjugate materials. For example, a recent study by Prof. Goodson and his colleagues proved that the thermal properties of CNTs can be remarkably modulated by encapsulation of fullerenes inside. ${ }^{9}$ On the other hand, Dr Kazu Suenaga et al. demonstrated that the electronic structure of single-walled CNTs can also be tuned through controlling the encapsulated $\mathrm{C}_{70}$ molecular orientations. ${ }^{10}$ Moreover, $\mathrm{AgBr}$ was proved to have a high stability to light irradiation after being encapsulated inside CNTs. ${ }^{11}$ Professor Xinhe Bao et al. designed a catalyst with iron nanoparticles confined inside pea-pod-like carbon nanotubes exhibiting a high activity and remarkable stability as a cathode catalyst. ${ }^{12}$ However, most of the incorporation studies and discussion on the nano-confinement phenomena have been performed on a scale of several nanometers, due to the small inner diameter of CNTs. ${ }^{13}$ Until now, there have been few research studies investigating the structure and behaviors of encapsulated materials on a larger scale up to micrometers. The effective scale of the nano-confinement phenomena remains unclear. Aiming to answer the above questions, we adopted carbon nanocones (CNCs) in this work, which are multilayered graphene-like carbon nanomaterials with a conical structure and an inner size continuously varying from several nanometers to micrometers. CNCs are regarded as a promising workhorse to study the filling phenomena and the crystallization behaviors of foreign materials on a nano-micron scale. 
CNCs are seamless and hollow graphene cones with five distinct apex angles, namely $19.2^{\circ}, 38.9^{\circ}, 60^{\circ}, 84.6^{\circ}$ and $112.9^{\circ}$, and were produced by pyrolysis of heavy oil in a carbon electric arc. ${ }^{\mathbf{1 4 , 1 5}}$ Due to their unique conical shape and high mechanical stability, CNCs can be used as a kind of novel atomic force microscopy tip. Abraham G. et al. have successfully encapsulated gold tips in CNCs via nanomanipulation to enhance the mechanical stability of the gold nanotips, which exhibit high plasticity limiting their application in some nanodevices. ${ }^{\mathbf{1 6}}$ Different from CNTs and SWCNHs, CNCs have a closed apex and the other side is open, which is suitable for incorporation experiments. The graphene-like structure of CNCs also provides a stable support for observing the encapsulated foreign materials using electron microscopy. Consequently, we successfully obtained Gd oxide nano-crystals that perfectly fit in the inner spaces of CNCs. The diameter of the conical Gd oxide crystals ranges from several nanometers to hundreds of nanometers. In addition, the conical Gd oxide crystals showed a high stability even at a temperature high enough to drive them apart from the apex of the CNCs.

\section{Experimental section}

The pristine CNCs were purchased from n-Tec AS. The CNC sample that was annealed at $2700{ }^{\circ} \mathrm{C}$ to improve the crystallinity of the material is denoted as CNCs- 1 in this paper. Gd acetate was purchased from J\&K Scientific company. Gd acetate $(5 \mathrm{mg})$ and CNCs-1 (5 mg) were dispersed and stirred in ethanol $(5 \mathrm{ml})$ at room temperature for $18 \mathrm{~h}$. The mixture was filtered with filter paper (the pore-size is $1 \mu \mathrm{m}$ ) and washed with ethanol ( $20 \mathrm{ml}, 1 \mathrm{ml}$ per time) 20 times to remove the excess Gd acetate outside CNCs-1 and some impurities to get GdAc@CNCs-1. After that, the sample was dried at $80{ }^{\circ} \mathrm{C}$ for $20 \mathrm{~h}$ in air, and then heated in a horizontal-chamber furnace at $700{ }^{\circ} \mathrm{C}$ under vacuum. In addition, we also used a CNC sample which was not annealed at $2700{ }^{\circ} \mathrm{C}$ (which is denoted as CNCs- 2 in this paper) to incorporate $\mathrm{Gd}$ acetate as a comparison.

The morphology and structure of the samples were studied by a transmission electron microscope (TEM) (Tecnai G2 F20UTWIN) and scanning transmission electron microscope (STEM), equipped for energy dispersive X-ray spectroscopy (EDX) and selected area electron diffraction (SAED). The TEM and STEM were operated at $200 \mathrm{kV}$. For the microscopic observations, the samples were firstly dispersed in ethanol by ultrasonication, and then dropped onto a porous carbon film-coated $\mathrm{Cu}$ grid.

\section{Results and discussion}

\subsection{Morphology and structure of encapsulated Gd compounds}

Compared to CNCs-1, which were annealed at a high temperature, CNCs-2 usually have many impurities (most of them are carbonaceous fragments) in the inner space close to the apex and the degree of graphitization is not as high as for CNCs- 1 . Meanwhile, CNCs-1 are very clean containing few impurities as shown in Fig. S1. $\dagger$ And the graphene layers in CNCs-1 are straight and neat. Fig. 1 shows six TEM images of CNCs-1 and
CNCs-2 with Gd compounds incorporated inside before and after heat treatment. Fig. 1a, b, and 1d, e are GdAc@CNCs-2 and GdAc@CNCs-1 before heat treatment, respectively, and Fig. 1c and $\mathrm{f}$ are Gd oxide encapsulated inside CNCs-2 and CNCs-1, respectively, after heat treatment at $700{ }^{\circ} \mathrm{C}$. The apex of the pristine CNCs was vacant inside (as presented in Fig. S1†), while a black area of Gd acetate clusters was found at the apex of the CNCs, as shown in Fig. 1. It is noteworthy that the incorporated Gd acetate clusters in CNCs- 1 and CNCs- 2 appeared in different structures. As seen in Fig. 1a and b, Gd acetate encapsulated inside CNCs-2 forms somewhere not far from the apex of CNCs2 , leaving the apex vacant. And a thin film of Gd acetate was formed adherent to the side wall of the CNCs-2 on a large scale. In contrast, Gd acetate clusters encapsulated inside the apex of CNCs-1 exist in a cone structure consistent with the inner shape of the CNCs-1 (Fig. 1d and e). This is the first time that conical nanomaterials formed by self-aggregation have been obtained in confined spaces. Their morphology is different from that of Gd compounds encapsulated inside CNTs in previous reports. Similarly, the Gd compounds formed inside SWCNHs were also spherical nanoparticles of a few nanometers, even though SWCNHs comprise conical nanohorns. ${ }^{17}$ Furthermore, the diameters of the encapsulated materials inside the CNTs and SWCNHs were only a few nanometers according to previous research studies, ${ }^{13}$ but the filling diameter of the $\mathrm{Gd}$ compounds inside the CNCs was up to hundreds of nanometers. The maximum filling diameter observed in our experiments was $428 \mathrm{~nm}$, and most of them fall in the range of 100$200 \mathrm{~nm}$ (the images are shown in Fig. S2 $\dagger$ ).

It is interesting to observe two different morphologies in the encapsulated Gd compounds inside the CNCs, and we can explain the different incorporation behaviors of the two kinds of CNCs by taking into account their structural features. As reported, the as-produced CNC sample consists of a mixture of carbon nanocones $(\sim 20 \%)$, carbon nanodiscs $(\sim 70 \%)$ and amorphous carbons, as well as some impurities $(\sim 10 \%)$ without post heat-treatment. ${ }^{18}$ The impurities may stop Gd acetate from
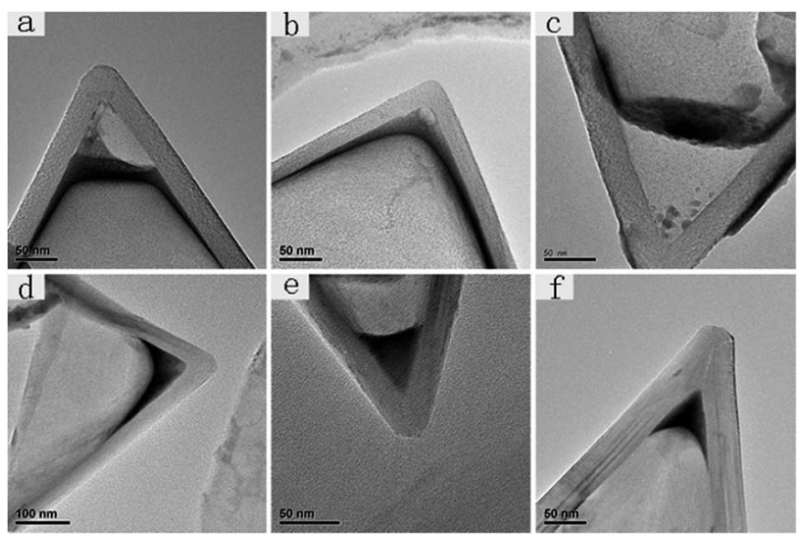

Fig. 1 TEM images of ( $a$ and b) CNCs-2 encapsulating Gd acetate before heat treatment, (c) CNCs-2 encapsulating Gd oxide after heat treatment, ( $d$ and e) CNCs-1 encapsulating Gd acetate before heat treatment, and (f) CNCs-1 encapsulating Gd oxide after heat treatment. 
entering the apex of CNCs-2, resulting in the morphology shown in Fig. 1a and b. In contrast, CNCs-1 treated at a high temperature have less obstacles to the incorporation of Gd acetate, and therefore we can observe cone-shaped Gd acetate incorporated in CNCs-1. On the other hand, the different chemical structures on the surfaces of CNCs-1 and CNCs-2 are an important reason resulting in their different encapsulation behaviors. Compared with CNCs-1, CNCs-2 are highly graphitized and contain few chemical groups on the surface. The different chemical environment in the interior of the CNCs affords different affinities between the encapsulated Gd compounds and the CNCs. It can be concluded that the chemical groups (mainly oxygencontaining functional groups) prevent Gd compounds from entering the apex of CNCs-2. This repelling effect is more apparent in a smaller space close to the apex of the CNCs-2. In contrast, the highly graphitized inner surface of CNCs-1 seems to have a high affinity to Gd compounds, resulting in a perfect conical shape of the encapsulated compounds fitting the inner space of the CNCs-1.

Furthermore, it is known that Gd acetate is transformed into Gd oxide by heat treatment above $650{ }^{\circ} \mathrm{C} .{ }^{19}$ For CNCs-2, it is clear to see that the $\mathrm{Gd}$ acetate clusters change into $\mathrm{Gd}$ oxide nanoparticles after heat treatment at $700{ }^{\circ} \mathrm{C}$ and some ultrafine Gd oxide nanoparticles successfully move to the apex of CNCs-2, although most of them still stay where they aggregated before (Fig. 1c). However, the Gd oxide retains the same cone-shaped structure and location as the Gd acetate clusters encapsulated in CNCs-1, as shown in Fig. 1f.

Fig. 2 presents high-resolution TEM (HRTEM) images of Gd oxide embedded inside CNCs-1 after heat treatment. Both single-crystal and polycrystal structural domains were observed. Fig. 2a and d are a TEM image and HRTEM image of a Gd oxide single-crystal, and Fig. 2b, c, and 2e, f are TEM images and HRTEM images of Gd oxide polycrystals. According to previous reports, the filling material usually has a small crystal domain size of less than $10 \mathrm{~nm}$ limited by the diameter of CNTs. ${ }^{\mathbf{2 0 , 2 1}}$

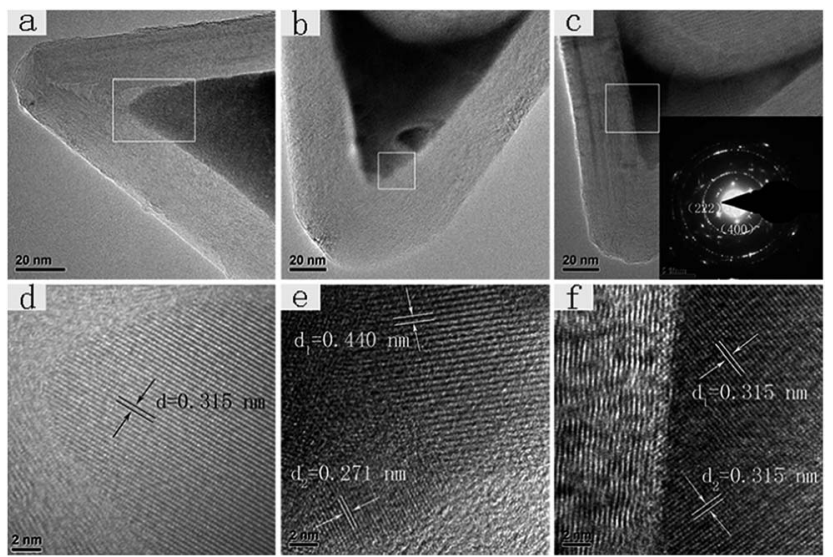

Fig. 2 TEM and HRTEM images of $\mathrm{Gd}_{2} \mathrm{O}_{3} \mathrm{QCNCS}-1$ : (a) TEM image of a $\mathrm{Gd}_{2} \mathrm{O}_{3} \mathrm{CCNC}-1$ with single-crystal $\mathrm{Gd}_{2} \mathrm{O}_{3}$; (b and c) TEM images of $\mathrm{Gd}_{2} \mathrm{O}_{3} \mathrm{aCNCs}-1$ with polycrystal $\mathrm{Gd}_{2} \mathrm{O}_{3}$; $(\mathrm{d}-\mathrm{f}$ ) HRTEM images of the designated areas in $(\mathrm{a}-\mathrm{c})$, respectively. The inset in $(c)$ is the corresponding SAED pattern of a $\mathrm{Gd}_{2} \mathrm{O}_{3} \mathrm{OCNC}-1$.
However, the size of the gadolinium oxide single-crystals growing inside CNCs-1 in our study can reach more than $50 \mathrm{~nm}$ laterally with an axial length of up to $72 \mathrm{~nm}$ (Fig. 2a and $\mathrm{S} 3 \dagger)$. And the sizes of the gadolinium oxide polycrystals are in the range from $50 \mathrm{~nm}$ to $400 \mathrm{~nm}$, among which the maximum size is up to $428 \mathrm{~nm}$ (as seen in Fig. $\mathrm{S} 2 \dagger$ ). The average values were 100-200 nm. The inter-lattice distance of the single-crystal Gd oxide in Fig. 2d was estimated to be $0.315 \mathrm{~nm}$. Meanwhile, three inter-lattice distances, i.e., $0.315 \mathrm{~nm}, 0.271 \mathrm{~nm}$ and $0.440 \mathrm{~nm}$, were found in the polycrystal Gd oxides in Fig. 2e and $\mathrm{f}$, which can be assigned to the layer spacing of cubic $\operatorname{Gd}_{2} \mathrm{O}_{3}$ (222), (400), and (211), respectively $\left(d_{(222)}=0.312 \mathrm{~nm}, d_{(400)}=\right.$ $\left.0.270 \mathrm{~nm}, d_{(211)}=0.441 \mathrm{~nm}\right)$. We also performed selected area electron diffraction (SAED) for an individual $\mathrm{Gd}_{2} \mathrm{O}_{3}$ @CNC-1, as shown in the inset of Fig. 2c. As a result, the spots indexed as (222) and (400) diffractions from cubic $\mathrm{Gd}_{2} \mathrm{O}_{3}$ were identified. According to the statistical results in our observations, most of the Gd oxide crystals encapsulated in CNCs-1 were polycrystals and only around 5\% of Gd oxide@CNCs- 1 were single-crystals. This is similar to previous studies in which both polycrystals and single-crystals usually co-exist in the composites of metals and CNTs, e.g. Au@CNTs, and Pt@CNTs. ${ }^{22}$ On the other hand, monocrystalline FeCo nanowires encapsulated inside CNTs were reported to be successfully produced by aerosol thermolysis. $^{23}$ However, the mechanism to control the preferential crystal structures of foreign materials in a confined space remains unclear. $^{24}$ Although single-crystals of encapsulated materials can be often observed on the scale of several nanometers, the growth direction and thus crystal morphology seemed to be easily disturbed during growth on a larger scale, resulting in polycrystals, as shown in our results.

Moreover, we performed elemental mapping of $\mathrm{Gd}_{2} \mathrm{O}_{3}$ @CNCs-1 under STEM, as shown in Fig. 3. It is clear to see that the material encapsulated inside the inner spaces was made of gadolinium (the green area) and oxygen (the yellow area) while

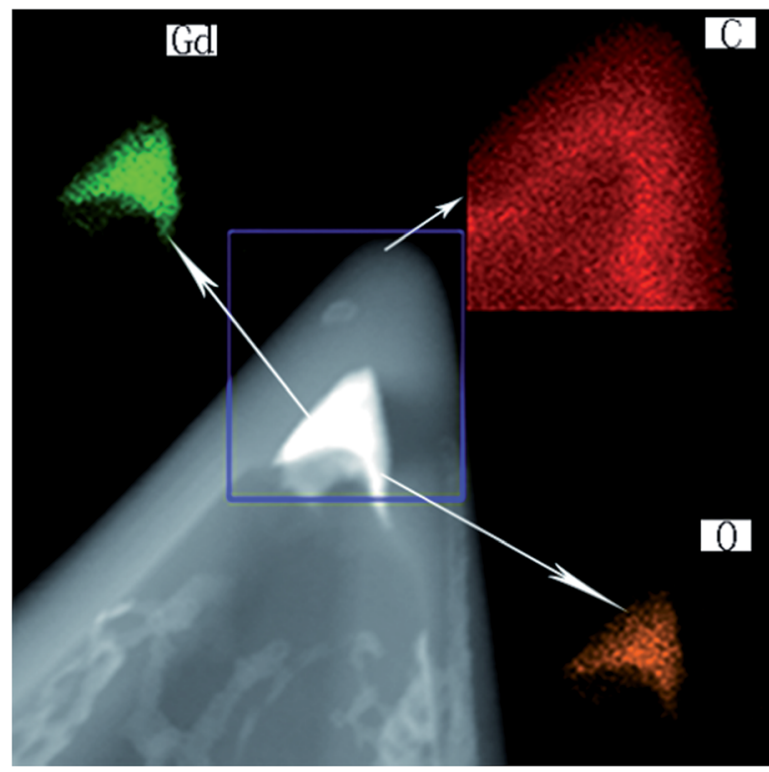

Fig. 3 The elemental mapping of $\mathrm{Gd}_{2} \mathrm{O}_{3} \mathrm{OCNC}-1$. 
the apex of the CNC was made of carbon (the red area). These results confirmed that the black material inside the apex of the CNC-1 was Gd acetate clusters and transformed into $\mathrm{Gd}_{2} \mathrm{O}_{3}$ crystals when heated at $700{ }^{\circ} \mathrm{C}$.

Consequently, we obtained Gd compounds encapsulated in CNCs showing various morphologies and structures. When using CNCs-1 as the filling template, we can get cone-shaped $\mathrm{Gd}_{2} \mathrm{O}_{3}$ crystals in a large size range up to hundreds of nanometers, which was not reported previously. This result indicates that the effective nano-confinement size can be around several hundreds of nanometers. The confined $\mathrm{Gd}_{2} \mathrm{O}_{3}$ crystals were mostly polycrystals; meanwhile, large single-crystal domains located at the apex of the CNCs were also observed. The size of the Gd oxide single-crystals in our study can reach more than $50 \mathrm{~nm}$ laterally with an axial length of up to $72 \mathrm{~nm}$. Our work demonstrates that CNCs can be used as a unique workhorse to study the crystallization behaviors of foreign materials in a confined space continuously from nanometers to micrometers.

\subsection{The filling percentage of CNCs with different apex angles}

To estimate the percentage of encapsulation, we randomly observed 110 CNCs-1 from the heat-treated sample under TEM. The statistical result is that 70 CNCs- 1 contained $\mathrm{Gd}_{2} \mathrm{O}_{3}$ nanocrystals, giving a filling percentage of $64 \%$. Since CNCs have five different apex angles $\left(19.2^{\circ}, 38.9^{\circ}, 60^{\circ}, 84.6^{\circ}, 112.9^{\circ}\right)$, the encapsulation yield is different for CNCs-1 with different apex angles. In order to determine the relationship between the apex angle of CNCs-1 and the filling ratio, we selected 10 CNCs-1 of each apex angle and calculated the filling ratio of the CNCs-1 with different apex angles. Three $\mathrm{Gd}_{2} \mathrm{O}_{3} @ \mathrm{CNC}-1$ samples were used for such calculations. As shown in Fig. 4, the filling ratio of CNCs with the apex angle of $38.9^{\circ}$ is the highest. Meanwhile, the CNCs with the apex angle of $112.9^{\circ}$ are difficult to fill. Generally, a CNC with a small apex angle is easier to fill than a large-angled CNC.

\subsection{The dynamics of cones encapsulating $\mathrm{Gd}_{2} \mathrm{O}_{3}$ under prolonged heating}

It has been proved above that the encapsulated $\mathrm{Gd}$ acetate decomposed into conical $\mathrm{Gd}_{2} \mathrm{O}_{3}$ crystals when heated at $700{ }^{\circ} \mathrm{C}$.

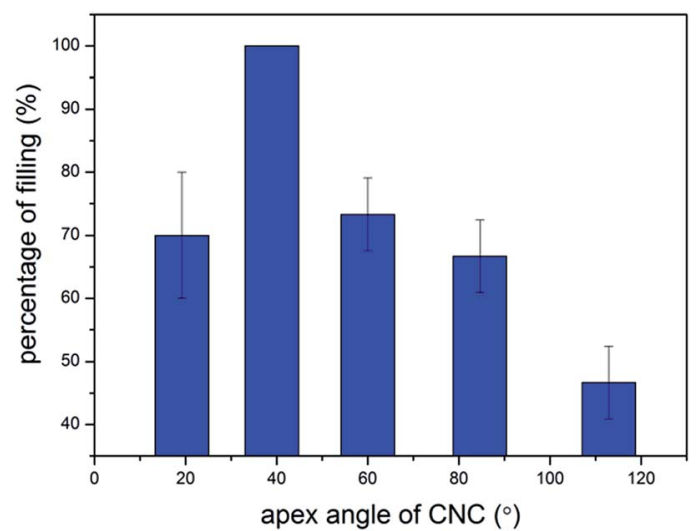

Fig. 4 The relationship between the apex angle of CNCs and the filling percentage of Gd compounds.
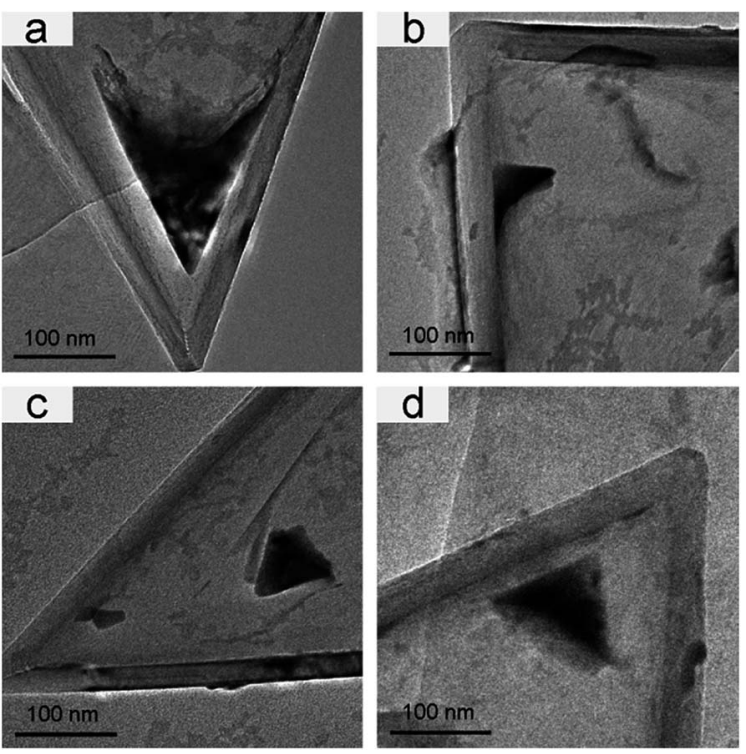

Fig. 5 TEM images of $\mathrm{Gd}_{2} \mathrm{O}_{3}$ nanocones moving apart from CNCs-1 after heat treatment at $700{ }^{\circ} \mathrm{C}$ for $2 \mathrm{~h}$ : (a and d) $\mathrm{Gd}_{2} \mathrm{O}_{3}$ nanocones started to separate with $\mathrm{CNCs}-1$; ( $b$ and $c$ ) $\mathrm{Gd}_{2} \mathrm{O}_{3}$ nanocones tended to move out of CNCs-1.

But the structure and behaviors of the $\mathrm{Gd}_{2} \mathrm{O}_{3}$ nanocones during a prolonged heat treatment were not clear. In our experiments after heating the samples at $700{ }^{\circ} \mathrm{C}$ for $2 \mathrm{~h}$, some interesting phenomena were observed.

As shown in Fig. 5, the $\mathrm{Gd}_{2} \mathrm{O}_{3}$ crystal started to separate from the apex of the CNC and had a tendency to move out of the CNC1 after heat treatment at $700{ }^{\circ} \mathrm{C}$ for $2 \mathrm{~h}$. The interesting thing was that the shape of the $\mathrm{Gd}_{2} \mathrm{O}_{3}$ crystal remained unchanged and was consistent with the apex of CNC-1. Thus, we obtained a cone-shaped $\mathrm{Gd}_{2} \mathrm{O}_{3}$ crystal and a vacant CNC-1. Referring to previous reports, $\mathrm{Gd}_{2} \mathrm{O}_{3}$ crystals encapsulated in the sheath of SWCNHs would move from the sheath to the center of the SWCNHs and aggregate forming a large spherical clumped crystal when heated at a high temperature. ${ }^{17}$ However in our study, the cone-shaped $\mathrm{Gd}_{2} \mathrm{O}_{3}$ crystal kept the same structure, even though it was driven to move outward from the CNC-1 during heating, indicating the high stability of the $\mathrm{Gd}_{2} \mathrm{O}_{3}$ cones. The morphology evolution and structural stability of the $\mathrm{Gd}_{2} \mathrm{O}_{3}$ cones inside CNCs under heating were also investigated in our in situ TEM observations, shown in Fig. S4. $\dagger$ In conclusion, from these preliminary results, it is possible to obtain cone-shaped target nanomaterials by taking advantage of this encapsulation-and-release feature of CNCs.

\section{Conclusions}

In summary, we investigated the encapsulation and the crystallization behaviors of Gd compounds in a confined nanospace using CNCs on a scale from nanometers to micrometers for the first time. Our results showed that the average crystal size of Gd oxide was over $100 \mathrm{~nm}$, and the maximum can be up to $428 \mathrm{~nm}$, which is much larger than the previously reported values. These results reveal that the nano-confinement effect can still 
function at a scale of hundreds of nanometers. Both singlecrystal and polycrystal $\mathrm{Gd}_{2} \mathrm{O}_{3}$ structures can form in the confined space. And the size of the single-crystals of Gd oxide can reach more than $50 \mathrm{~nm}$ laterally with an axial length of up to $72 \mathrm{~nm}$. Moreover, the thus-formed Gd oxide nanocones kept the same cone structure after being heated at a high temperature, implying high stability. The novel Gd oxide nanocones, as well as the filled CNCs, may find some new applications due to their new structures, such as in functional SPM probes, nanocatalysis etc. Our work also demonstrates that CNCs can be used as a unique workhorse to study the structure and behaviors of foreign materials in a confined space continuously from nanometers to micrometers.

\section{Conflicts of interest}

There are no conflicts to declare.

\section{Acknowledgements}

This work was financially supported by the Ministry of Science and Technology of China (2016YFA0203200), the National Thousand Talent Program of China, and the National Science Foundation of China (51472095, 51672093, 51602112, and 51602097).

\section{Notes and references}

1 S. lijima, Nature, 1991, 354, 56-58.

2 S. Iijima, M. Yudasaka, R. Yamada, S. Bandow, K. Suenaga, F. Kokai and K. Takanashi, Chem. Phys. Lett., 1999, 309, 165-170.

3 A. K. Geim, K. S. Novoselov, S. V. Morozov, D. Jiang, Y. Zhang, S. V. Dubonos, I. V. Grigorieva and A. A. Firsov, Science, 2004, 306, 666-669.

4 A. C. Dillon, K. M. Jones, T. A. Bekkedahl, C. H. Kiang, et al., Nature, 1997, 386, 377-379.

5 W. Xu, K. Takahashi, Y. Matsuo, Y. Hattori, M. Kumagai, S. Ishiyama, K. Kaneko and S. Iijima, Int. J. Hydrogen Energy, 2007, 32, 2504-2512.
6 R. Yuge, M. Yudasaka, J. Miyawaki, Y. Kubo, T. Ichihashi, H. Imai, E. Nakamura, H. Isobe, H. Yorimitsu and S. Iijima, J. Phys. Chem. B, 2005, 109, 17861-17867.

7 A. Hashimoto, et al., Proc. Natl. Acad. Sci. U. S. A., 2004, 101, 8527-8530.

8 K. Ajima, M. Yudasaka, T. Murakami, A. Maigne, K. Shiba and S. Iijima, Mol. Pharm., 2005, 2, 475-480.

9 T. Kodama, M. Ohnishi, W. Park, T. Shiga, J. Park, T. Shimada, H. Shinohara, J. Shiomi and K. E. Goodson, Nat. Mater., 2017, 16, 892-897.

10 S. Okubo, T. Okazaki, K. Hirose-Takai, K. Suenaga, S. Okada, S. Bandow and S. Iijima, J. Am. Chem. Soc., 2010, 132, 1525215258.

11 K. Kobayashi, K. Suenaga, T. Saito, H. Shinohara and S. Iijima, Adv. Mater., 2010, 22, 3156-3160.

12 D. Deng, L. Yu, X. Chen, G. Wang, L. Jin, X. Pan, J. Deng, G. Sun and X. Bao, Angew. Chem., 2013, 52, 371-375.

13 J. Miyawaki, M. Yudasaka, H. Imai, H. Yorimitsu, H. Isobe, E. Nakamura and S. Iijima, J. Phys. Chem. B, 2006, 110, 5179-5181.

14 M. Ge and K. Sattler, Chem. Phys. Lett., 1994, 220, 192-196.

15 A. Krishna, M. M. J. Treacy, J. Hugdahl, et al., Nature, 1997, 388, 451-454.

16 A. G. Cano-Marquez, et al., Sci. Rep., 2015, 5, 10408.

17 R. Yuge, T. Ichihashi, J. Miyawaki, T. Yoshitake, S. Iijima and M. Yudasaka, J. Phys. Chem. C, 2009, 113, 2741-2744.

18 S. N. Naess, A. Elgsaeter, G. Helgesen and K. D. Knudsen, Sci. Technol. Adv. Mater., 2009, 10, 065002.

19 G. A. M. Hussein, J. Phys. Chem., 1994, 98, 9657-9664.

20 D. Zhou, S. Seraphin, J. Jiao, J. C. Withers and R. Loutfy, Appl. Phys. Lett., 1993, 63, 2073-2075.

21 Y. Lu, Z. Zhu and Z. Liu, Carbon, 2005, 43, 369-374.

22 A. Govindaraj, B. C. Satishkumar, M. Nath and C. N. R. Rao, Chem. Mater., 2000, 12, 202-205.

23 A. L. Elías, J. A. Rodríguez-Manzo, et al., Nano Lett., 2005, 5, 467-472.

24 F. Banhart, N. Grobert, M. Terrones, J. C. Charlier and P. M. Ajayan, Int. J. Mod. Phys. B, 2001, 15, 4037-4069. 